\title{
SULFURIZATION OF SINTERED REDUCED IRON WITH HYDROGEN SULFIDE AND ITS OVERALL RATE DESCRIPTION BY VOLUME REACTION MODEL
}

\author{
SHOICHI KIMURA, TETSUO MORITA, \\ SETSUuI TONE AND TSUTAo OTAKE \\ Department of Chemical Engineering, \\ Osaka University, Toyonaka, Osaka 560
}

\begin{abstract}
The sulfurization of sintered reduced iron in a hydrogen gas stream containing hydrogen sulfide was investigated. To describe the behavior of this reaction system accompanied by significant decrease in porosity of reacting solid, the volume reaction model was modified in due consideration of the variation of effective diffusivity. Observed overall reaction rate under the influence of diffusion limitation was well represented by this model. The intrinsic rate constant was then separated from the overall rate data and correlated in an Arrhenius-type equation. The rate process is also discussed in detail in relation to the shrinking core model and the accuarcy of its application is dealt with.
\end{abstract}

\section{Introduction}

Reaction between a gas and a solid is frequently encountered in the process industry. For each gassolid reaction a knowledge of the rate of reaction is a prerequisite to the analysis of a process.

To describe the behavior of gas-solid reactions, a number of models have been proposed. Among them, two distinct models can be specified. The model in which the reaction is considered to take place uniformly throughout a porous particle is called the "volume reaction model" (VRM) $)^{5}$. The model which treats the reaction as narrowly confined to the reaction interface between the reactant solid and the product is known as the "shrinking core model" (SCM) Both models have been successfully applied to extensive sorts of reaction system ${ }^{8,14,17}$.

In this work, the sulfurization of reduced iron with hydrogen sulfide is dealt with. This reaction is considered the basic one in the desulfurization of hot coal gas by means of iron oxide sorbents ${ }^{3,18}$. VRM is modified in due consideration of structural change of solid since this reaction is accompanied by a significant change in molar volume of solid between the reactant and the product. The rate of reaction is also discussed in relation to SCM.

Received February 6, 1981. Correspondence concerning this article should be addressed to S. Kimura. T. Morita is now with Suntory Ltd., Nirasaki, Yamanashi 407.

\section{Experiments}

\section{1 Solid materials}

Reactant solid is a sintered mixture of reduced iron and inert silica. The original solid is composed of reagent-grade ferric oxide $\left(\mathrm{Fe}_{2} \mathrm{O}_{3}\right)$ and silica powder $\left(\mathrm{SiO}_{2}\right)$, which are mixed and blended with a proper amount of water, dried at $100^{\circ} \mathrm{C}$ for 10 hours and then sintered at $1200^{\circ} \mathrm{C}$ for 3 hours. The sintered pellets were crushed and screened for use as sample particles for reduction. The obtained particles of desired size were exposed in a stream of hydrogen gas diluted with inert nitrogen at the temperature of the following sulfurization reaction.

\subsection{Experimental procedures}

A thermal gravitational analyzer was utilized to obtain conversion against time data. Iron oxide particles of about $50 \mathrm{mg}$ were placed in a monolayer in the platinum basket and first reduced by hydrogen gas. When a decrease of sample weight was no longer detected, hydrogen sulfide was added to the reduction gas and the sulfurization of the reduced iron was carried out.

Reaction temperature was kept constant through the entire course of both reduction and sulfurization. Experimental conditions are listed in Table 1. The velocity of the reactant gas stream in the reactor tube was kept in the range in which gas film resistance was negligibly small. 


\begin{aligned} & Table 1 Experimental conditions \\ & Reaction temperature : $457-704^{\circ} \mathrm{C} \\ &$ Gaseous reactant \\ & Hydrogen sulfide $: 0-0.52 \mathrm{vol} \% \\ &$ Hydrogen $: 75 \mathrm{vol} \% \\ &$ Nitrogen $:$ balance \\ & Solid reactant $: 41.2 \mathrm{wt} \%$ of Fe \\ & Particle radius $: 0.09-0.5 \mathrm{~mm} \\ &$\hline\end{aligned}

Table 2 Physical properties of solid materials

\begin{tabular}{lccc} 
Material & $\begin{array}{c}\text { Reaction } \\
\text { temp. }\left[{ }^{\circ} \mathrm{C}\right]\end{array}$ & $\begin{array}{c}\text { BET surface } \\
\text { area }\left[\mathrm{m}^{2} / \mathrm{g}\right]\end{array}$ & $\begin{array}{c}\text { Porosity } \\
\varepsilon_{0} \& \varepsilon^{\prime}[-]\end{array}$ \\
$\mathrm{Fe} / \mathrm{SiO}_{2}$ & 515 & 2.5 & 0.352 \\
& 543 & 2.7 & 0.357 \\
& 635 & 2.4 & 0.365 \\
& 704 & 2.2 & 0.350 \\
$\mathrm{FeS}_{x} / \mathrm{SiO}_{2}$ & 515 & 0.73 & 0.122 \\
& 543 & 0.61 & 0.130 \\
& 635 & 0.67 & 0.135 \\
& 704 & 0.53 & 0.134 \\
\hline
\end{tabular}

Porosity and internal surface area of solid materials were measured by a mercury porosimeter and by BET with nitrogen as adsorption gas, respectively.

\section{Change of Solid Structure Resulting from the Reaction}

The sulfurization of the reduced iron is considered to proceed according to

$$
\mathrm{Fe}+x \mathrm{H}_{2} \mathrm{~S} \rightarrow \mathrm{FeS}_{x}+x \mathrm{H}_{2}
$$

The ultimate weight decrease in the reduction of iron oxide sample agreed well with the stoichiometric prediction. In the sulfurization of the reduced iron, nonstoichiometric iron sulfide was produced. Its average composition was observed to be $\mathrm{FeS}_{1.04}$ independently of reaction conditions by the ultimate weight increase and chemical analysis of sulfur content ${ }^{12}$.

Physical properties of the reduced iron and the iron sulfide obtained at several temperature levels are listed in Table 2. Very low BET surface area indicates that the pores of both solids are mainly macro pores.

It is obvious that the porosity decreases considerably as a result of sulfurization. Since each solid has similar porosity in the temperature range investigated, it may be averaged to be 0.356 for the reduced iron and 0.130 for the iron sulfide, respectively. The sulfurization of the reduced iron reduces its porosity by $63.5 \%$. This is greater than the extent predicted by the following molar volume difference between the two solids.

The molar volume difference gives the porosity of iron sulfide as

$$
\varepsilon=\varepsilon_{0}-\left(\frac{1}{x} v_{\mathrm{Fe} \mathrm{S}_{x}}-v_{\mathrm{Fe}}\right) C_{B 0}
$$

$C_{B 0}$ represents moles of reduced iron initially contained per unit solid volume and is $0.0147 \mathrm{~mol} / \mathrm{cm}^{3}$ for the present case. Approximating the molar volume of $\mathrm{FeS}_{x}$, that is, $v_{\mathrm{FeS}_{x}}$, by that of FeS, Eq. (2) predicts that $\varepsilon=0.204$. Consequently, one may understand that $36.2 \%$ of the pores are removed in addition to the mere swelling of solid due to molar volume increase. This is considered to result from the pore interconnection which occurs essentially in the same manner as sintering ${ }^{7}$.

In modeling the behavior of the present reaction system, the structural change described above must be taken into consideration.

\section{Mathematical Modeling}

\section{1 VRM with variable effective diffusivity}

This section develops a VRM modified to account for the change in solid structure. Expressing the gassolid reaction taking place in a spherical porous particle by the general stoichiometry:

$$
\mathrm{A}(\text { gas })+b \mathrm{~B} \text { (solid) } \rightarrow p \mathrm{P} \text { (solid) }+q \mathrm{Q} \text { (gas) }
$$

Taking account of the depletion of the solid reactant, a simple power law rate expression may be introduced to represent the rate equation ${ }^{1,8,15,17)}$. Thus, the consumption rate of gaseous reactant A per unit volume of reacting solid is given by

$$
-r_{A}=-\frac{1}{V} \frac{d N_{A}}{d t}=k C_{A} C_{B}
$$

where the first order in each gas and solid reactant is postulated ${ }^{12)} . C_{B}$ is defined by moles of B contained per unit volume of reacting solid. $k$ is the rate constant based on unit solid volume, which is assumed constant during the course of reaction.

The following assumptions are also employed to simplify the model.

1) The system is under isothermal conditions.

2) Pseudo-steady state approximation is valid.

3) Gas-film resistance is negligibly small.

4) Particle radius remains constant.

Differential material balance equations with respect to the gas and solid reactants can then be written in nondimensional formulae, respectively, as

$$
\begin{gathered}
\frac{1}{\lambda^{2}} \frac{\partial}{\partial \lambda}\left(f \lambda^{2} \frac{\partial y}{\partial \lambda}\right)+\phi^{2} \frac{\partial z}{\partial \theta}=0 \\
-\frac{\partial z}{\partial \theta}=y z
\end{gathered}
$$

with the boundary and initial conditions:

$$
\begin{array}{ll}
y=1 & \text { at } \lambda=1 \\
\frac{\partial y}{\partial \lambda}=0 & \text { at } \lambda=0 \\
z=1 & \text { at } \theta=0
\end{array}
$$

where $y=C_{A} / C_{A 0}, z=\mathrm{C}_{B} / C_{B 0}, \lambda=r / R$, and $\theta=b k C_{A 0} t$. $f=D_{e} / D_{e 0}$ and represents the variation of the effective diffusivity. Thiele-type modulus $\phi$ is defined on the basis of initial effective diffusivity $D_{e 0}$ as

$$
\phi=R \sqrt{\frac{k C_{B 0}}{D_{e 0}}}
$$


Let us here define the effectiveness factor for gassolid reactions to measure the diffusion effect ${ }^{\theta}$ :

$$
\eta=\frac{\text { actual reaction rate }}{\text { reaction rate obtainable under the }} \begin{aligned}
\text { conditions of the bulk gas stream }
\end{aligned}
$$

Using average concentration of the reactant solid contained in a particle, $\eta$ can be given by

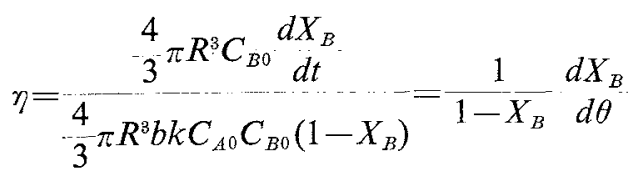

where $X_{B}$ is the average conversion given by

$$
X_{B}=1-3 \int_{0}^{1} \lambda^{2} z d \lambda
$$

With known effectiveness factor, the intrinsic reaction rate constant can then be obtained by the observed overall reaction rate as

$$
k=-\frac{1}{b C_{A 0} \eta\left(1-X_{B}\right)}\left(\begin{array}{c}
d X_{B} \\
d t
\end{array}\right)_{\text {obs. }}
$$

\section{2 Variation of effective diffusivity}

It is assumed that the solid structure is represented by the porosity of reacting solid. Then its change is accounted for through the effective diffusivity, which for a porous solid is correlated with its porosity by ${ }^{16)}$

$$
f=\frac{D_{e}}{D_{0 e}}=\left(\frac{\varepsilon}{\varepsilon_{0}}\right)^{m}
$$

where $m$ lies between 2 and 3. $D_{e}$ signifies the effective diffusivity for a solid with porosity of $\varepsilon$. $\varepsilon_{0}$ is the initial value of $\varepsilon$.

According to the random pore model of Wakao and $\mathrm{Smith}^{13)}, m$ reduces to 2 . However, when there is pore interconnection as in the present system, the tortuosity factor for diffusion takes a much larger value than can be accounted for by the random pore model alone $e^{7,11}$. As described previously, it is considered that $36.2 \%$ of pores are removed by interconnection. Taking account of this on the increase in the tortuosity factor, $m$ can be approximated by 3 for the present system.

If it is assumed that the porosity is linearly dependent on the local extent of solid conversion ${ }^{10)}$, we have

$$
\varepsilon=\varepsilon^{\prime}+\left(\varepsilon_{0}-\varepsilon^{\prime}\right) z
$$

where $\varepsilon^{\prime}$ is the porosity of the final product solid. Substituting Eq. (15) into Eq. (14) gives the relative effective diffusivity $f$ as a function of $z$,

$$
f=\frac{D_{e}}{D_{e 0}}=\left[\frac{\varepsilon^{\prime}}{\varepsilon_{0}}+\left(1-\frac{\varepsilon^{\prime}}{\varepsilon_{0}}\right) z\right]^{3}
$$

Now, solutions of Eqs. (5) and (6) under their relevant boundary conditions can be obtained numerically by approximating them in difference equations. The implicit form of the difference equation was applied in the computation procedures ${ }^{2)}$.

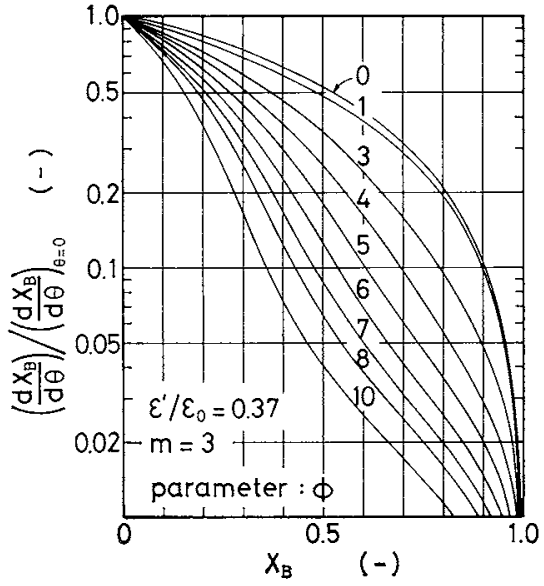

Fig. 1 Ratio of fractional conversion rate at any time to initial rate

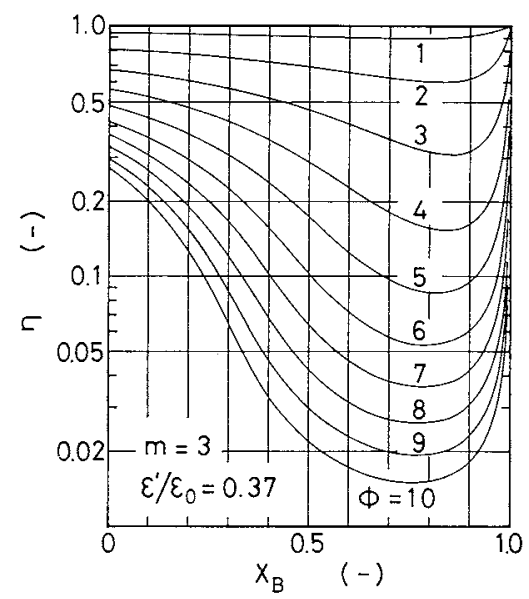

Fig. 2 Effectiveness factor to give magnitude of diffusion effect

$\varepsilon^{\prime} / \varepsilon_{0}$ in Eq. (16) takes a constant value for each reaction system and is $0.37 \mathrm{in}$ the present case. Hence, Thiele-type modulus $\phi$ is the only parameter in the model and the application of the model prediction begins with its estimation.

Figure 1 shows the ratio of the nondimensional conversion rate at any time to the initial value calculated by the model for each $\phi$. Although the conversion rate by the model is obtained in terms of nondimensional time $\theta$, this can be eliminated by taking the ratio as the ordinate in Fig. 1. Then, the conversion against the actual process time data are the only prerequisites to utilizing this chart. Thus, superimposing the observed ratio of conversion rate $\left(d X_{B} / d t\right) /\left(d X_{B} / d t\right)_{t=0}$ on this chart gives an estimate of $\phi$ by the best fit with the model.

Figure 2 provides the effectiveness factor theoretically calculated for each value of $\phi$. The effectiveness factor needed for the calculation of the rate constant $k$ can be evaluated at any extent of conversion with the known value of $\phi$ determined with the aid of Fig. 1. 


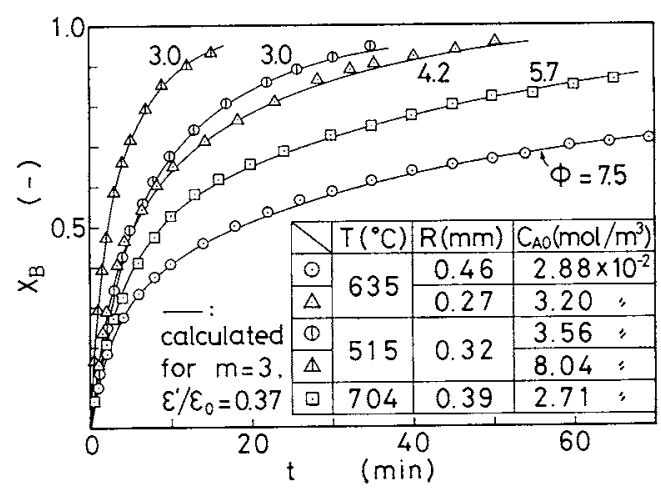

Fig. 3 Progress of reaction and fit with model prediction

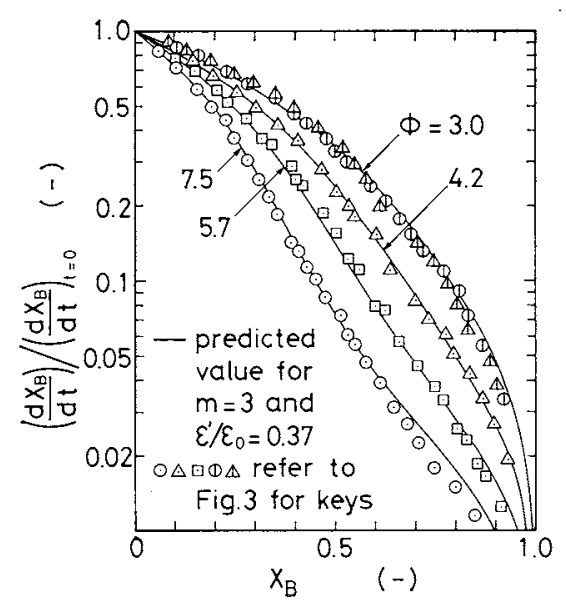

Fig. 4 Estimation of $\phi$ by superimposing observed data on Fig. 1

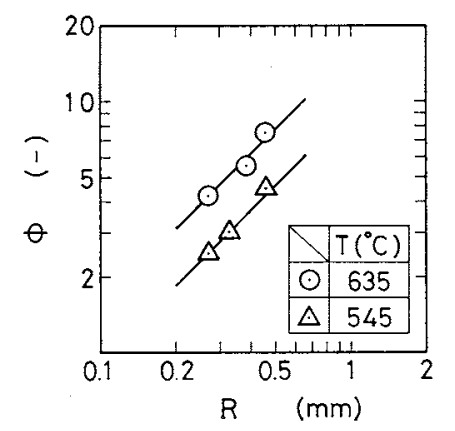

Fig. 5 Test of model based on definition of $\phi$

\section{Analysis of Data Based on the Model}

\section{1 Applicability of the model}

Several examples of the progress of sulfurization under various reaction conditions are shown in Fig. 3 as conversion against time data. Since the product iron sulfide takes nonstoichiometric composition, the extent of conversion was calculated by

$$
X_{B}=\frac{\text { increase of sample weight at any time }}{\text { ultimate increase in sample weight }}
$$

Graphical differentiation of each series of conversion data depicted in Fig. 3 provides sets of $d X_{B} / d t$ vs. $X_{B}$. Superimposing these data on Fig. 1 according to the

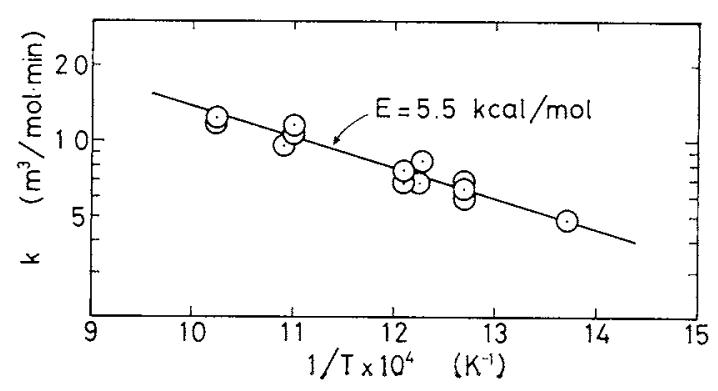

Fig. 6 Arrhenius plot of intrinsic rate constant

manner described above gives an estimate of $\phi$ for each run as is shown in Fig. 4.

In general, a decrease of conversion rate was observed near the very end of reaction. This is considered to result from the change in effective reacting surface area of solid phase ${ }^{17)}$. In this work, however, the slight discrepancy of observed data from the model calculation during the final stage of the reaction, say $X_{B}>0.9$, was neglected.

One may understand from Fig. 4 that the value of $\phi$ giving the best fit with data is independent of gaseous reactant concentration, which is consistent with the model prediction. On the contrary, $\phi$ takes distinct values for different particle sizes, which requires firstorder dependence on particle radius as is obvious by its definition. The test of the model on this point is shown in Fig. 5, where $\phi$ evaluated for each different particle size is plotted against its radius. The proportionality of $\phi$ to $R$ is satisfactory. In conclusion, the present model can be accepted for its proper description of the behavior of the present system.

\section{2 The intrinsic reaction rate constant}

The estimated magnitude of $\phi$ implies that the observed rate data include the diffusion limitation ${ }^{8)}$. Hence, with known value of $\phi$, the effectiveness factor must be evaluated by Fig. 2. Observed overall conversion rate is then substituted into Eq. (13) to calculate the intrinsic reaction rate constant $k$ separately from the diffusion effect.

The Arrhenius plot of the rate constant evaluated at $X_{B}=0.5$ in this manner is shown in Fig. 6. The slope of the straight line gives an apparent activation energy of $5.5 \mathrm{kcal} / \mathrm{mol}$. Westmoreland et al. ${ }^{19}$ reported $5.69 \mathrm{kcal} / \mathrm{mol}$ for the sulfurization of $\mathrm{MnO}$, $7.20 \mathrm{kcal} / \mathrm{mol}$ for $\mathrm{ZnO}$, and $8.83 \mathrm{kcal} / \mathrm{mol}$ for $\mathrm{V}_{2} \mathrm{O}_{3}$. Hasatani et al. ${ }^{4}$ also obtained $1.5 \mathrm{kcal} / \mathrm{mol}$ for the sulfurization of unsintered reduced iron. Hence, the observed activation energy in this work is much closer to the values of Westmoreland. The intrinsic rate constant is then correlated by

$$
k=217 \mathrm{e}^{-5500 / R T}
$$

Since Eq. (17) predicts $k$ at any temperature, the nondimensional time $\theta$ can be converted into the actual process time by $t=\theta / b k C_{A 0}$. The model pre- 
diction now can be compared with the observed data on the conversion-against-time diagram as solid lines in Fig. 3. The model calculations are in satisfactory agreement with the data.

\section{3 Approximation of the present model by SCM}

When $f=D_{e} / D_{e 0}<1$ as in the present system, the reacting solid becomes less porous as the reaction progresses. Thiele-type modulus based on the effective diffusivity for the completely converted solid $D_{e}^{\prime}$ can be calculated by $\phi^{\prime}=\left(D_{e 0} / D_{e}^{\prime}\right)^{1 / 2} \phi$. Hence, in the present system we have $\phi^{\prime}=4.4 \phi$. Since $\phi^{\prime}$ is 4.4 times greater than $\phi$, one can anticipate that the reaction rate will be controlled by the diffusion process in the product layer even for a small value of the original Thiele type modulus $\phi$.

To visualize this phenomenon, the conversion data in Fig. 3 are plotted on the basis of the following equation for SCM in the product layer diffusion control regime. Thus, in terms of the nondimensional parameters associated with the present $\operatorname{model}^{9}$,

$$
\begin{aligned}
\left(\frac{6 b C_{A 0} D_{e}^{+}}{C_{B 0} R^{2}}\right) t & =\frac{6 \theta}{\left(\phi^{+}\right)^{2}} \\
& =1-3\left(1-X_{B}\right)^{2 / 3}+2\left(1-X_{B}\right)
\end{aligned}
$$

Where $D_{e}^{+}$is the apparent effective diffusivity for the product layer hypothetically formed from the exterior of a particle inward. $\phi^{+}$is Thiele-type modulus based on this diffusivity.

As is observed in Fig. 7, the data show the linear relationships as the reaction sufficiently progresses. Based on Eq. (18), the slope of the straight portion gives $\phi^{+}$. This value is then plotted against $\phi^{\prime}=4.4 \phi$ in Fig. 8. The two values of $\phi^{+}$and $\phi^{\prime}$ become closer as $\phi^{\prime}$ increases. This fact shows that diffusion through the product solid comes to dominate the rate process.

Figure 9 shows a comparison of the present model with SCM based on the effectiveness factor. The effectiveness factor for SCM is calculated by combining Eqs. (11) and (18). The value of $\phi^{+}$needed in this calculation is obtained by the straight line approximation shown in Fig. 7. When $\phi$ becomes as large as 20 , the present system can be approximated widely, say $X_{B}>0.5$ within $10 \%$ error, by SCM. The diffusion rate controlling the rate process in this case is close to that in the entire product solid.

\section{Conclusion}

The volume reaction model for gas solid reactions was modified in due consideration of the structural change in solid phase, where the effective diffusivity was shown to vary with the porosity change of reacting solid. Two working diagrams were then constructed for the model utilization.

The model parameter $\phi$, Thiele-type modulus, can be determined by observed overall conversion rate data

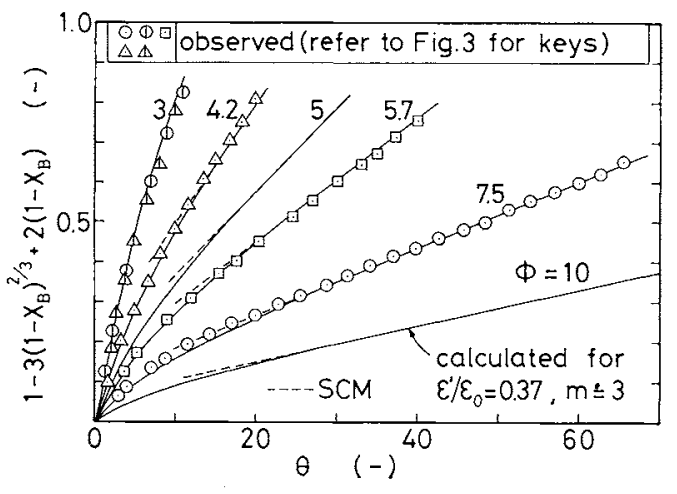

Fig. 7 Approximation of model by SCM

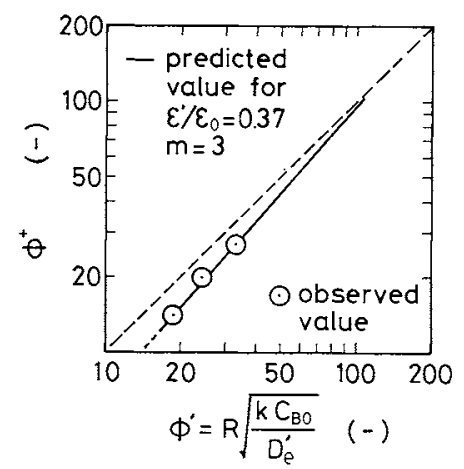

Fig. 8 Approach of effective diffusivity toward that of final product solid

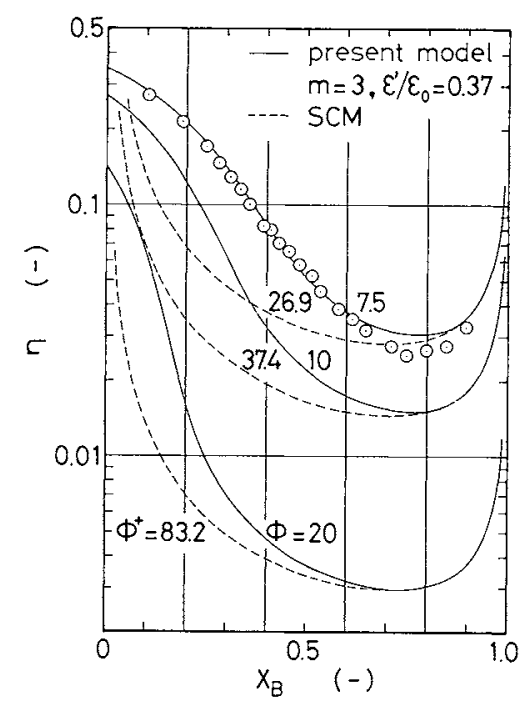

Fig. 9 Comparison of model with SCM based on effectiveness factor

from Fig. 1. Figure 2 then provides the effectiveness factor for $\phi$ determined by Fig. 1. This factor separates the intrinsic rate constant from the overall reaction rate.

The model prediction was successfully applied to the sulfurization of sintered reduced iron in a hydrogen stream containing hydrogen sulfide accompanied by a significant porosity decrease. The intrinsic rate constant was evaluated by the overall rate data and cor- 
related in an Arrhenius-type equation.

\section{Nomenclature}

$b=$ stoichiometric coefficient in Eq. (3)

$C_{A} \quad=$ local gaseous reactant concentration in a particle

$C_{A 0} \quad=$ gaseous reactant concentration in the bulk gas stream

$C_{B} \quad=$ local solid reactant concentration in a particle

$D_{e} \quad=$ effective diffusivity for reacting solid

$E \quad=$ apparent activation energy

$=$ relative diffusivity defined by $D_{e} / D_{e 0}$

$=$ reaction rate constant per unit volume of reacting solid

$\left[\mathrm{m}^{3} / \mathrm{mol} \cdot \mathrm{min}\right]$

$\begin{array}{ll}m & =\text { index in Eq. (14) } \\ N_{A} & =\text { moles of gaseous reactant A }\end{array}$

$R \quad=$ radius of a particle

$R \quad=$ ideal gas law constant

$-r_{A} \quad=$ reaction rate per unit volume of reacting solid

$\left[\mathrm{mol} / \mathrm{m}^{3} \cdot \mathrm{min}\right]$

$t \quad=$ process time $\quad[\mathrm{min}]$

$=$ volume of reacting solid $\quad\left[\mathrm{m}^{3}\right]$

$v \quad=$ molar volume of solids specified with subscripts

$\left[\mathrm{m}^{3} / \mathrm{mol}\right]$

$X_{B} \quad=$ average extent of conversion $\quad[--]$

$x \quad=$ nonstoichiometric coefficient in Eq. (1) [-]

$y \quad=C_{A} / C_{A 0}$

$z \quad=C_{B} / C_{B 0}$

$\varepsilon \quad=$ porosity of reacting solid $\quad[-]$

$\phi \quad=$ Thiele-type modulus defined by Eq. (10) [-]

$\lambda \quad=r / R \quad[-]$

$\eta \quad=$ effectiveness factor $\quad[-]$

$\theta \quad=$ nondimensional time defined by $\theta=b k C_{A 0} t \quad[-]$

$\langle$ Subscripts〉

$0 \quad=$ initial value

$\langle$ Superscripts $\rangle$

$=$ values for the completely converted solid
$=$ values for the hypothetically formed product layer from the exterior of a particle inward

\section{Literature Cited}

1) Boersma, M. A. M., J. A. M. Spierts and H. S. van der Baan: Chem. Eng. Sci., 35, 1237 (1980).

2) Carnahan, B., H. A. Luther and J. O. Wilkes: "Applied Numerical Methods", John Wiley, N. Y. (1969).

3) Farrior, W. L., A. M. Poston and E. C. Oldaker: Preprint for the Fourth Energy Resources Conference, Kentucky, 1976.

4) Hasatani, M., M. Yuzawa, S. Sugiyama and C. Y. Wen: Kagaku Kogaku Ronbunshu, 6, 515 (1980).

5) Ishida, M. and C. Y. Wen: AIChE J., 14, 311 (1968).

6) idem: Chem. Eng. Sci., 23, 125 (1968).

7) Kim, K. K. and J. M. Smith: AIChE J., 20, 670 (1974).

8) Kimura, S., J. Nakagawa, S. Tone and T. Otake: J. Chem. Eng. Japan, 14, 190 (1981).

9) Levenspiel, O.: "Chemical Reaction Engineering", John Wiley, N. Y. (1972).

10) Levenspiel, O.: "The Chemical Reactor Omnibook", OSU Book Stores, Inc., Oregon (1979).

11) Ramachandran, P. A. and J. M. Smith: Chem. Eng. J., 14, 137 (1977).

12) Morita, T.: Master's Thesis, Osaka University, Osaka (1980).

13) Wakao, N. and J. M. Smith: Chem. Eng. Sci., 17, 825 (1962).

14) Weisz, P. B. and R. D. Goodwin: J. Catal., 2, 397 (1963).

15) idem: ibid., 6, 227 (1966).

16) Wen, C. Y.: Ind. Eng. Chem., 60 (9), 34 (1968).

17) Wen, C. Y. and N. T. Wu: AIChE J., 22, 1012 (1976).

18) Westmoreland, P. R. and D. P. Harrison: Envi. Sci. Tech., 10, 659 (1976).

19) Westmoreland, P. R., J. B. Gibson and D. P. Harrison: ibid., 11, 488 (1977).

(Presented at the 14th Autumn Meeting of the Society of Chemical Engineers, Japan.) 